\title{
Should my accountant sing La Traviata at the Met?
}

\author{
Bostjan Seruga, MD; Malcolm Moore, MD
}

$\mathrm{T}$ he request for this Point / Counterpoint was received on Sept. 6, 2007. It was also the day that Italian opera star Luciano Pavarotti died at the age of 71 from advanced pancreatic cancer. One year earlier, Pavarotti had developed resectable pancreatic cancer and underwent a pancreaticoduodenectomy, or Whipple resection, in New York. Why did he have his surgery in New York? Well, Pavarotti was a well-informed man and was aware of evidence that surgical morbidity and mortality are markedly better if this operation is done in a larger volume centre that has appropriate surgical expertise and postoperative support. As part of the evidence-driven movement to improve quality and safety of patient care, leaders within surgical oncology argue appropriately that complex oncological surgery should be restricted to highly trained surgical oncologists in larger academic centres. While the evidence is probably best in hepatobiliary surgery, there are similar data for some of the more difficult urological surgical procedures, such as cystectomy and retroperitoneal lymph node dissection. ${ }^{1,2}$ We suspect that if any of the CUAJ readers were to develop a resectable urological cancer, they would seek to have their operation performed by the best surgeon in a centre with first-rate postoperative care.

Given the importance of training, expertise and appropriate supportive care is so clearly understood by surgical oncologists, it is curious that some leaders in urological surgery would then throw these principles out the window and suggest that urologists be able to order chemotherapy. As medical oncologists rather than psychiatrists, we are not properly trained to comment on the cognitive processes that might lead intelligent and otherwise rational individuals to take such a point of view. Rather, we will indicate why it is not appropriate for urologists to deliver systemic therapy.

Surgery is the oldest treatment modality for cancer and the majority of patients who survive their cancers do so because of surgical resection of the primary. Medical oncology is a much younger specialty, born in the aftermath of World War II when drugs like nitrogen mustard were used to treat malignant lymphoma. It is now approaching middle age and 2008 represents the 35th anniversary of the formal recognition of medical oncology as a subspecialty by the American Board of Internal Medicine. ${ }^{3}$ It is a rapidly evolving field, moving from the empirical toward tailored treatment. Some notable achievements came in the late 1970s when effective treatment with combined systemic chemotherapy was developed for advanced testicular cancer. $^{4,5}$ The regimens developed, improved and evaluated by medical oncologists over the following 2 decades assured constantly improving care such that most patients with advanced disease are now cured. Such treatment is complex, and among medical oncologists it is recognized that patients with testicular cancer should be treated by those with experience and expertise. Is it possible that urologists could achieve similar results if they were to treat testicular cancer patients with chemotherapy? We do not need clinical trials to answer this question. The relation between potential for cure and serious toxicity is finely balanced and at least equivalent to that associated with a major operation; it requires comparable training and experience. Similar logic applies to chemotherapy used for other urological cancers. From the perspective of quality of care and patient safety, only a medical oncologist with appropriate training and expertise in the systemic treatment of early and advanced urological cancer can give the best possible care to these patients. Physicians and surgeons benefit both themselves and their patients if they are self-critical, recognize their own limitations and allow those who are best qualified to treat the patient to do so.

What is best for the patient should always be given priority over what is best for the doctor. Patient safety is the dominant factor. A urological oncologist and a medical or radiation oncologist trained in urological cancer should understand when surgery, radiation therapy and chemotherapy are appropriate 
in the management of urological cancers. It is true that using standard algorithms for dose based on weight and height, the urologist, or anyone with a basic mathematical background, could calculate and "order" the first cycle of chemotherapy for a good performance patient with urological cancer who has no significant comorbidities. However, acute toxicity with nausea, vomiting, renal insufficiency and myelosuppression during treatment with chemotherapy is a stressful and fearful event for every patient. Management of toxicity is evolving rapidly and we can now ameliorate many of the disturbing side effects of chemotherapy and improve quality of life. Myelosuppression can lead to febrile neutropenia that can be serious or fatal and should be treated vigorously. Knowledge about optimal antibiotic therapies and supportive care is necessary to benefit patients and save lives. Neurotoxicity and pulmonary toxicity are common chronic toxicities of drugs used to treat urological cancer. Also, while some of the newer targeted agents have a different toxicity profile than standard cytotoxic therapies, they cause a broad array of cutaneous, cardiovascular and gastrointestinal effects that are sometimes life-threatening and can be a major therapeutic challenge. Knowledge of pharmacokinetics is crucial for understanding the destiny of toxic drugs in the organism and how changes in the function of different organs caused by intercurrent illness, the cancer or the drugs being used can impair the processes of metabolism and excretion and increase the chance of irreversible and disabling toxicity. It is critical to effectively manage toxicities and maintain the dose intensity of chemotherapy during the treatment. Decisions about dose and schedule in the setting of toxicity and organ dysfunction require judgment that comes from experience and appropriate training in internal medicine and medical oncology. Do surgeons have enough training, interest and time to address all these important issues? Would patients have continuous care and support while surgeons are performing timeconsuming and exhausting surgeries? Is the surgical ward the best place for a patient with postchemotherapy complications?

Beyond the fact that it will compromise outcome and impair patient safety, there is a second important reason that urologists should not give chemotherapy: it is disrespectful, both to the patients they treat and to their colleagues. Cancer therapy has improved markedly in the past decade because of multidisciplinary interactions both at the individual patient level and in collaborative clinical research. For urologists to think that they can administer chemotherapy without the background of medical training or the full-time commitment or support structures of their medical oncology colleagues is both discourteous and naive.

We conclude by returning to Pavarotti. On the stage, an excellent performance seems effortless, but its essence lies in countless hours of rehearsal behind the curtain, well-hidden from the world. Whom do we want to see on stage when the curtain is raised and we have purchased an expensive entrance ticket to the opera? Although we have an accountant friend who sings very capably, it is Pavarotti who will give the best performance and fulfill our expectations. It is implausible that surgeons or other health professionals can fill the roles of medical oncologists while performing the complex opera entitled "systemic treatment of cancer." Neither the audience nor the patients deserve a second-rate performance.

The prescription that says that "urologists should give chemotherapy" is a defective remedy and should not be filled. If the leadership of urological surgery in Canada wishes to improve patient care in urological cancer, it can best do so by advocating for more of the complex surgical procedures to be done under their expert hands.

From the Division of Medical Oncology and Hematology, Princess Margaret Hospital, University Health Network, University of Toronto, Toronto, Ont.

The positions provided in the Point/Counterpoint series are presented as general information and do not necessarily reflect the personal opinions of the authors.

This article has been peer reviewed.

Competing interests: None declared.

\section{References}

1. Davoli M, Amato L, Minozzi S, et al. Volume and health outcomes: an overview of systemic reviews. Epidemiol Prev 2005;29:3-63.

2. Nuttall $M$, van der Meulen J, Phillips $N$, et al. A systematic review and critique of the literature relating hospital or surgeon volume to health outcomes for 3 urological cancer procedures. J Urol 2004;172:2145-52.

3. Davidson NE. The maturation of medical oncology. Lancet Oncol 2007;8:57-8.

4. Einhorn $L H$, Williams SD. The role of cis-platinum in solid-tumor therapy. $N$ Engl J Med 1979;300:1295-7.

5. Williams SD, Birch R, Einhorn $L H$, et al. Treatment of disseminated germ cell tumors with cisplatin, bleomycin, and either etoposide or vinblastine. N Engl J Med 1987;316: $1435-40$.

Correspondence: Dr. Bostian Seruga; Bostian.Seruga@uhn.on.ca 